\title{
NOVEL CYCLOMATRIX-TYPE POLYPHOSPHAZENE MICROSPHERES CROSSLINKED WITH OCTACHLOROCYCLOTETRAPHOSPHAZENE: PREPARATION AND CHARACTERIZATION
}

\author{
Yasemin SÜZEN ${ }^{1, *}$, Simge METINOĞLU ÖRÜM ${ }^{1,2}$ \\ ${ }^{1}$ Department of Chemistry, Faculty of Science, Anadolu University, Yunusemre Campus, Eskişehir, Turkey \\ 2 Department of Chemistry, Faculty of Science and Arts, Mehmet Akif Ersoy University, İstiklal Campus, Burdur, Turkey
}

\begin{abstract}
In this article, highly cross-linked inorganic and organic hybrid polyphosphazene microspheres including amino groups on the surface have been successfully obtained via self-assembly polycondensation approach using 4,4'-diaminodiphenylmethane and octachlorocyclotetraphosphazene which is a new crosslinker. Experiments were performed without adding a surfactant or stabilizer, using only an ultrasonic bath. Otherwise, effect of different molar ratios of the reactants, reaction time and ultrasonic power were investigated on preparing morphologically good spheres. The reactivity of octachlorocyclotetraphosphazene was investigated comparing with hexachlorocyclotriphosphazene which is the most popular crosslinker cyclic phosphazene molecule. The size of acquired microspheres were approximately same, and $1.00 \mu \mathrm{m}$. The novel microspheres were characterized by SEM, FTIR, ZETA, UV, Fluorescense, XRD and TGA methods.
\end{abstract}

Keywords: Octachlorocyclotetraphosphazene, 4, 4'-diaminodiphenylmethane, Microspheres, Self-assembly, Polyphosphazene

\section{INTRODUCTION}

Recently, synthesis, characterization and application studies of cyclomatrix polyphosphazene nano/microspheres via precipitation polymerization technique have accelerated [1-8]. This technique is basic, rapid and has some advantages such as no need to any stabilizer or surfactant molecules.

Up to now, hexachlorocyclotriphosphazene ( $\mathrm{HCCP}, \mathrm{N}_{3} \mathrm{P}_{3} \mathrm{Cl}_{6}$ ) has been used as a crosslinker to obtain cyclomatrix type polyphosphazene spheres [9-10]. It is the most popular and widely studied molecule in polyphosphazene studies [11-15]. However, there are very few studies about octachlorocyclotetraphosphazene (OCCP, $\mathrm{N}_{4} \mathrm{P}_{4} \mathrm{Cl}_{8}$ ) eight-membered ring, has skeletal flexibility due to its $\mathrm{K}$ and $\mathrm{T}$ conformations [16-20]. OCCP has eight chlorine atoms which are bound to phosphorus atoms, can react with amine or hydroxyl groups by nucleophilic substitution reaction and it is more reactive than HCCP [21]. OCCP is a better crosslinker than HCCP since it has more chlorine atoms and flexible structure. HCCP is not flexible and nearly planar [20]. So, the crosslinking between molecules of HCCP is more difficult than in the molecules of OCCP.

In present study, for the first time, we used octachlorocyclotetraphosphazene (OCCP) as a crosslinker and synthesized novel inorganic-organic hybride crosslinked polyphosphazene microspheres, poly(cyclotetraphosphazene-co-4,4'diaminodiphenylmethane (OCDA-MS), containing amine groups on the surface, by one pot self-assembly polycondensation. When octachlorocyclotetraphosphazene (OCCP) was used as a crosslinker, the reaction time was short and reaction did not require darkness, water addition or temperature control, and use of any stabilizer or surfactant [15, 22-24].

This is the first time use of OCCP as a crosslinker in precipitation polymerization. Obtained cyclomatrix polyphosphazene nano/microspheres with OCCP, can be used in many applications such as drug delivery and controlled release systems, catalytic researches, some immobilization and adsorption studies etc. as 
used in crosslinked polyphosphazene microspheres obtained from hexachlorocyclotriphosphazene [5,25-27]. Thus, this approach would be a pioneer research study for the future works in this field.

\section{EXPERIMENTAL}

\subsection{Materials and Methods}

Octachlorocyclotetraphosphazene (OCCP) (Otsuka Chemical Co., Ltd.) and hexachlorocyclotriphosphazene (HCCP) (Sigma-Aldrich Company) were purified by fractional crystallization from dry n-hexane. 4,4'-diaminodiphenylmethane (DADPM) ( $\geq 97.0 \%$, GC), triethylamine (TEA) ( $\geq 99.0 \%$ ) acetonitrile (anhydrous,99.8 \%), tetrahydrofuran (THF) (anhydrous, $\geq 99.9 \%$ ) and ethanol were purchased from Sigma-Aldrich Company and were used as received.

Scanning Electron Microscopy (SEM) measurements were run on a ZEISS Ultra Plus (ZEISS ULTRA 55, APOLLO XP3) electron microscope. The samples were coated with gold before SEM observations. Fourier Transform Infrared Spectroscopy (FTIR, Perkin Elmer Spectrum 100 spectrometer) were used to explain the functional groups on the structure of the microspheres. Ultraviolet-visible (UV-vis) absorbance spectra were measured on a Shimadzu UV-3150 spectrometer. The fluorescent spectra measurements were performed on a Varian Cary Eclipse Fluorescence spectrophotometer. Dynamic Light Scattering (DLS) measurements were performed with Malvern ZEN 3600 Instruments. X-ray diffraction (XRD) pattern was recorded by using a Bruker AXS, S8 TIGER Advance instrument equipped with $\mathrm{Cu} \mathrm{K} \alpha$ radiation performed at $40 \mathrm{kV}$ and $40 \mathrm{~mA}$. The TGA measurements were carried out using platinum crucibles by PerkinElmer Diamond TG/DTA Thermal Analysis Instrument in the static air atmosphere with heating rate $10 \mathrm{~K} \mathrm{~min}^{-1}$ in the temperature range of $40-900{ }^{\circ} \mathrm{C}$.

\subsection{Synthesis of the OCDA-MS Microspheres}

Octachlorocyclotetraphosphazene, OCCP, $(100 \mathrm{mg}, 0.2157 \mathrm{mmol})$, as a crosslinker, was dissolved in 20 $\mathrm{ml}$ acetonitrile in a $100-\mathrm{ml}$ flask. The solution of 4,4'-diaminodiphenylmethane, DADPM, (42.7 mg, $0.2157 \mathrm{mmol})$ in $10 \mathrm{ml}$ acetonitrile was added to OCCP solution. The mixture was stirred in an $(100 \mathrm{~W}$, $53 \mathrm{kHz})$ ultrasonic bath for 15 minutes, at $40{ }^{\circ} \mathrm{C}$. Then, TEA $(1.5 \mathrm{ml}, 10.78 \mathrm{mmol})$, as an acid acceptor was added to the reaction medium and the reaction was continued for 3 hours (Scheme 1). The precipitated white polyphosphazene microspheres were isolated by centrifugation $(5000 \mathrm{rpm}, 30 \mathrm{~min})$, washed with THF, distilled water and ethanol, respectively. Finally, the powder product was dried under vacuum at $50{ }^{\circ} \mathrm{C}$. The same procedure was used for other mol ratios of OCCP and DADPM (Table 1).

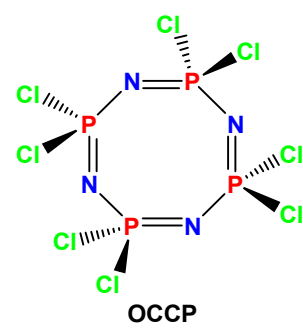<smiles>Nc1ccc(Cc2ccc(N)cc2)cc1</smiles>

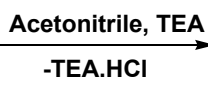

Scheme 1. Synthesis reaction of OCDA-MS

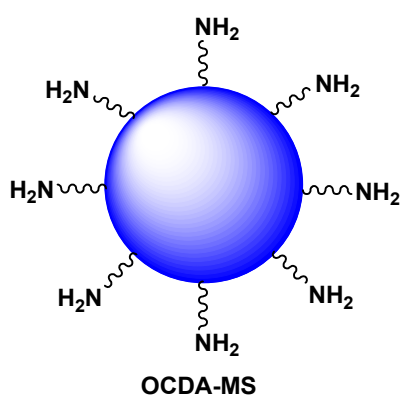

Table 1. Composition of microspheres at constant OCCP amount

\begin{tabular}{ccccc}
\hline Molar ratio(OCCP:DADPM) & OCCP $(\mathrm{mg})$ & DADPM $(\mathrm{mg})$ & TEA $(\mathrm{ml})$ & Acetonitrile $(\mathrm{ml})$ \\
\hline $1: 1$ & 100 & 42.7 & 1.5 & 30 \\
$1: 2$ & 100 & 85 & 1.5 & 30 \\
$1: 3$ & 100 & 128 & 1.5 & 30 \\
$1: 4$ & 100 & 171 & 1.5 & 30 \\
\hline
\end{tabular}




\subsection{Reactivity Study of OCCP}

The two parallel reactions were performed using OCCP and HCCP as crosslinkers and were used 1:1, OCCP:DADPM-HCCP:DADPM molar ratios by the same experimental procedure, explained in Section 2.2 (Scheme 2) As can be seen from Figure 1, OCDA-MS started to precipitate within 5 minutes. However, there were no precipitation during the 3 hours when HCCP is used as a crosslinker. The reaction was left in a dark place for $72 \mathrm{~h}$ for the completion of the precipitation.

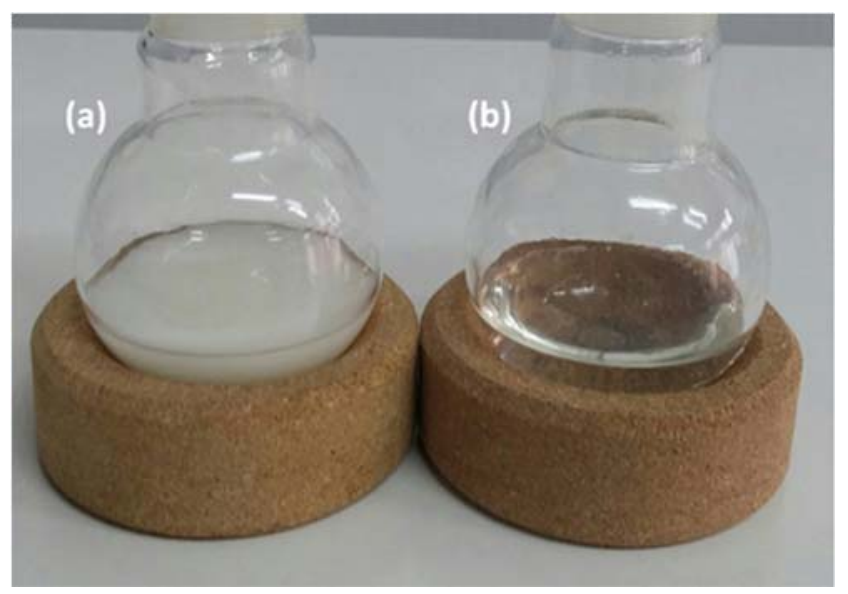

Figure 1. The appearance of the reaction media used a) OCCP b) HCCP after 5-min ultrasonication

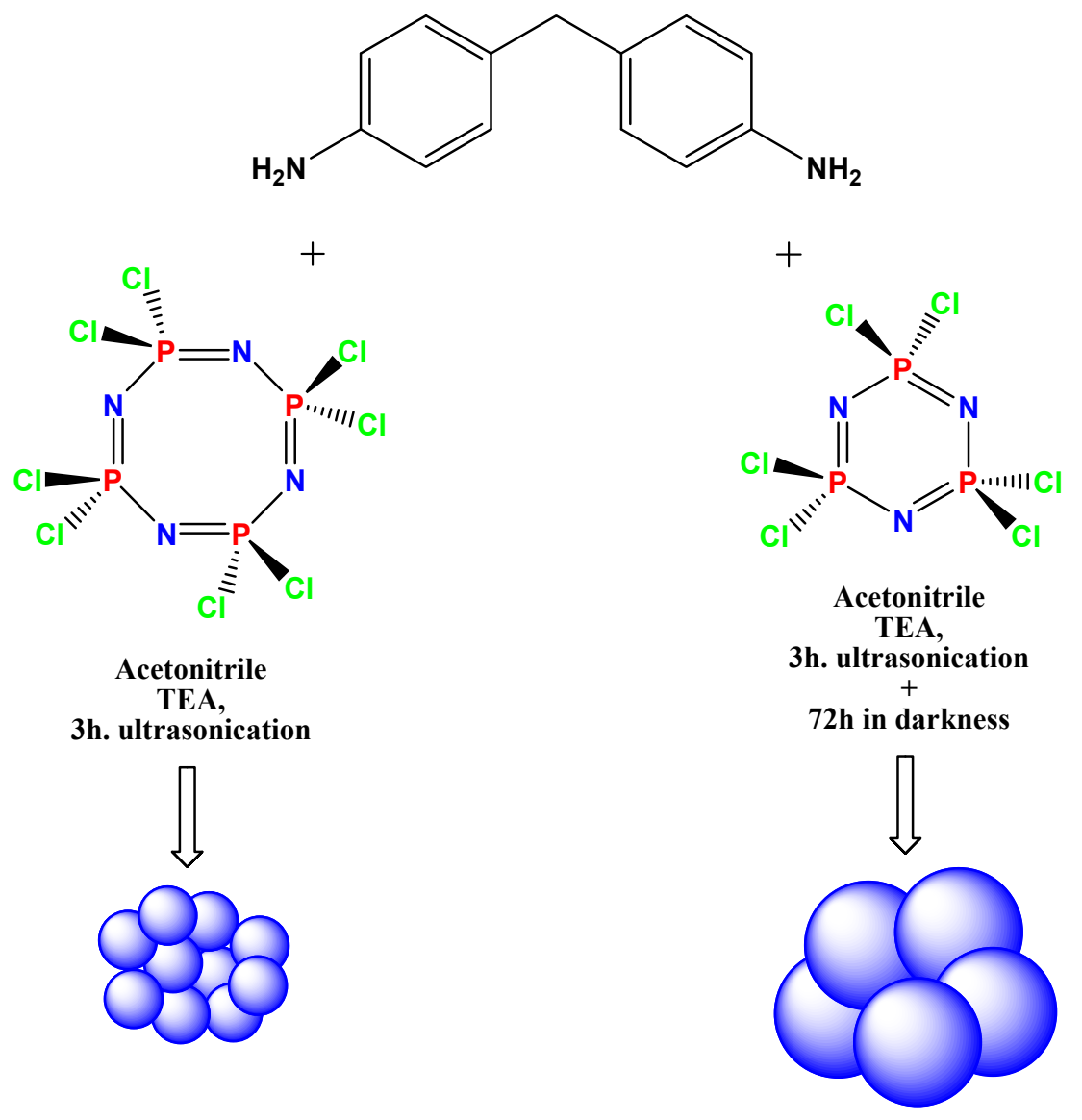

Scheme 2. The comparison of OCCP and HCCP in precipitation polymerization reaction 


\section{RESULTS AND DISCUSSION}

\subsection{Preparation, Surface Morphology and Size Distribution of OCDA-MS}

The synthesis of OCDA-MS was achieved successfully via a one-pot and basic polymerization technique. In polymerization reaction of OCCP with DADPM, OCCP is a new crosslinker molecule having eight chlorine atoms and a flexible ring. These features provide high cross-linking ability to OCCP. Besides, TEA is an acid acceptor which forms TEA.HCl salt during the reaction. Thus, it is thought that, continuously formation of this salt have accelerated the nuclephilic substitution reaction between OCCP and DADPM (Scheme 3) [24, 28, 33].

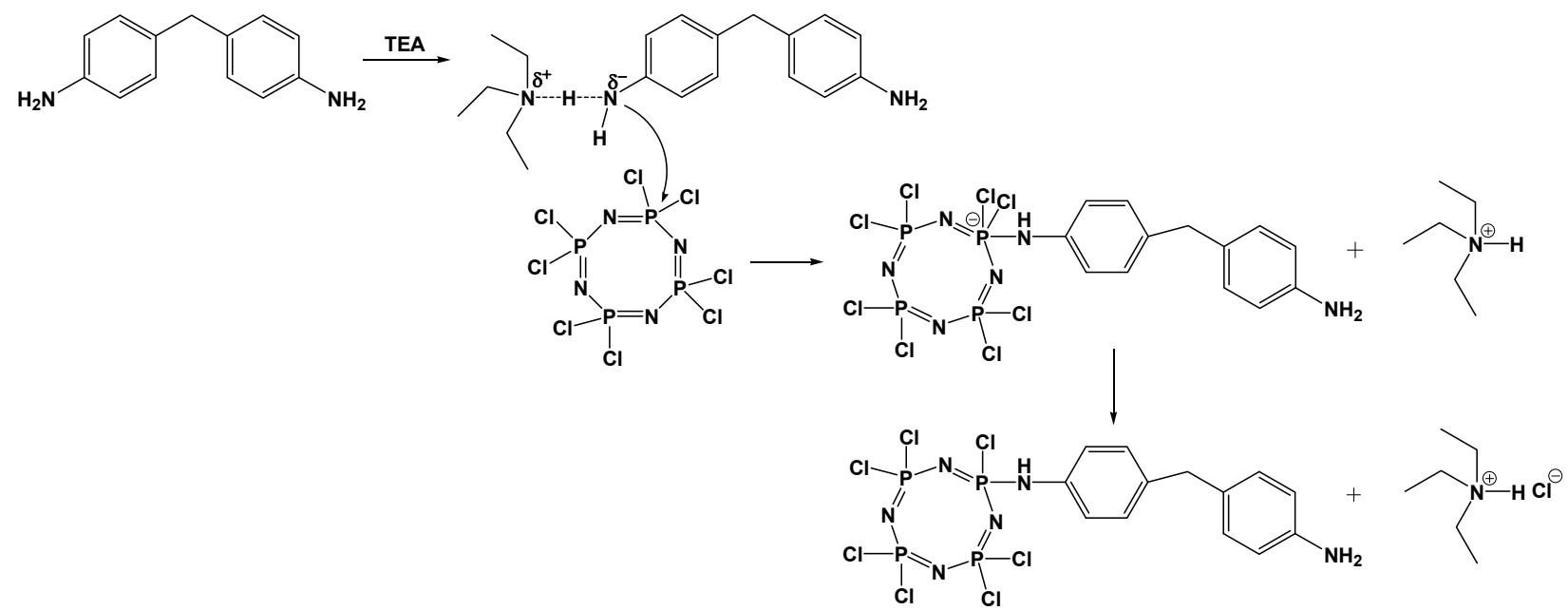

Scheme 3. The reaction pathway of OCCP, DADPM and TEA in acetonitrile

The self-assembly formation mechanism of cyclomatrix type polyphosphazene microspheres, crosslinking with octachlorocyclotetraphosphazene (OCCP) is similar with the suggested mechanism when the hexachlorocyclotriphosphazene (HCCP) used as crosslinker [2,9,29,30]. At first step of selfassembly polycondensation reaction, oligomers are obtained by reaction between monomer (DADPM) and crosslinker (OCCP). Then, oligomers aggregate together to form primary nucleus particles. After that stage, primary stable particles are generated due to the aggregation of the primary nucleus particles with each other by hydrogen bonds. As soon as the stable particles are generated, the particles grow in size by absorbing oligomeric species instead of primary particles. Hence, microspheres (OCDA-MS) acquired at the end of the polymerization, don't have pores inside, Scheme 4. 


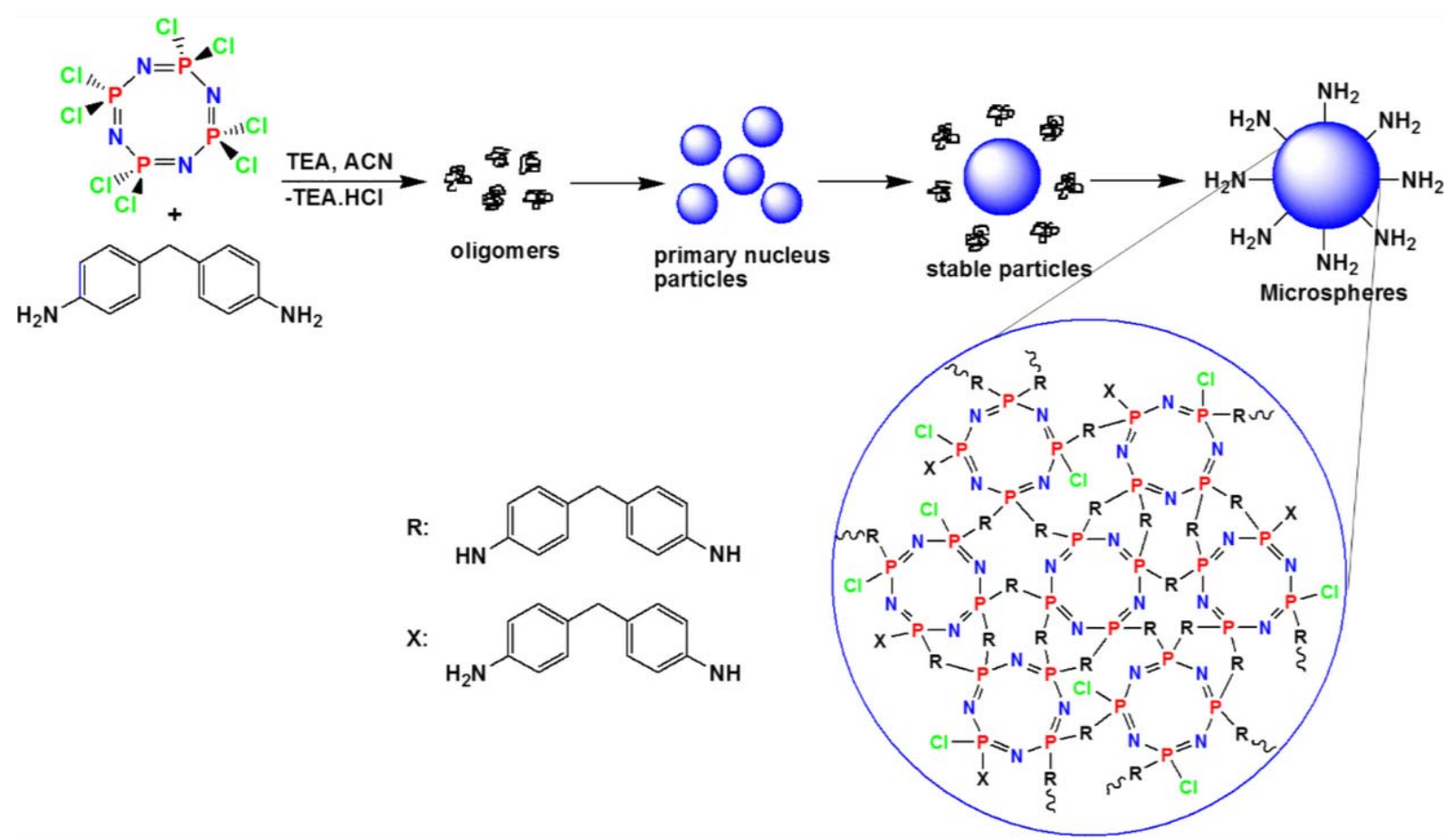

Scheme 4. Self-assembly polycondensation mechanism of cyclomatrix polyphosphazene microspheres

SEM images of synthesized microspheres revealed effect of changing OCCP and DADPM ratios on formation of microspheres (Figure 2). Particle size distribution of obtained microspheres with different OCCP:DADPM molar ratios were given in Figure 3. SEM images (Figure 2a) showed that synthesized microspheres using 1:1 (OCCP:DADPM) molar ratio, approximately have same size, $1.0 \mu \mathrm{m}$ and were morphologically smooth spheres. On the contrary, it was determined from SEM images, when especially 1:3 and 1:4 molar ratios (OCCP:DADPM) were used, microspheres were different by means of particle sizes. It might be due to changing in amount of DADPM, OCCP and TEA in reaction. During the reaction, the excess amount of DADPM remains in the reaction medium, OCCP and TEA are restrictive for the formation of the microspheres. Thus, oligomeric species are not obtained at the same sizes. So, primary nucleus particles and finally obtained microspheres can have different sizes (Figure $2 \mathrm{c}$ and $2 \mathrm{~d}$ ). Then, obtained OCDA-MS were first washed with THF to remove the unreacted OCCP and DADPM. Then, TEA.HCl salt was eliminated washing with distilled water. Finally, OCDA-MS were washed with ethanol for fending off water from microspheres. 


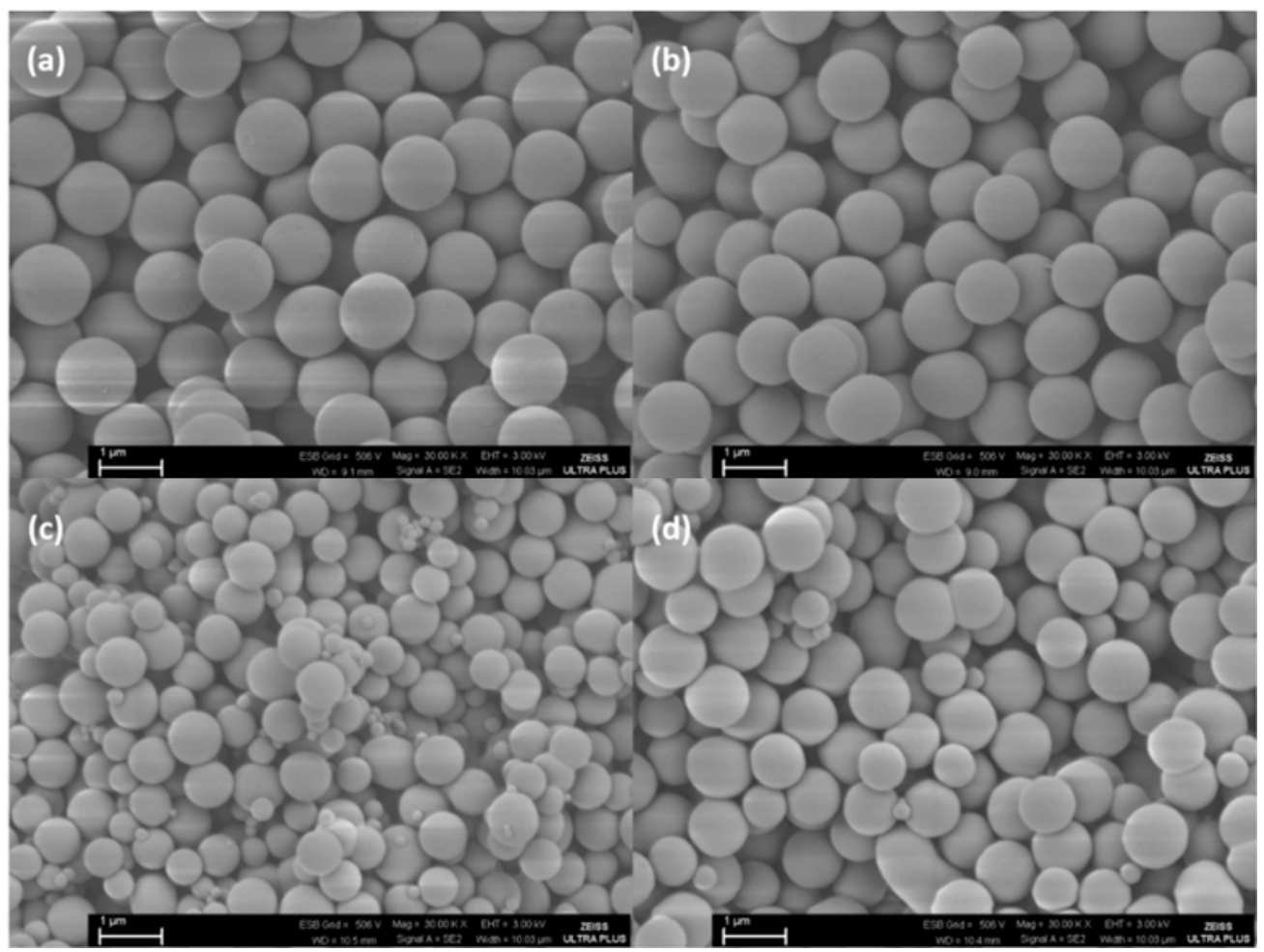

Figure 2. SEM images of spheres obtained using different molar ratios of OCCP:DADPM a) 1:1 b) 1:2 c) 1:3 d) 1:4 OCCP:DADPM

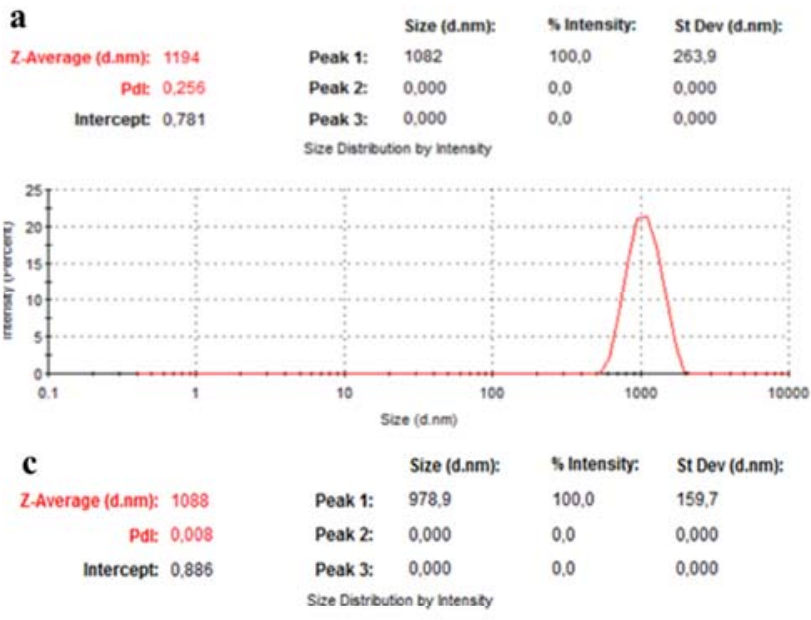

\begin{tabular}{|c|c|c|c|c|c|}
\hline b & & & Size (d.nm): & S Intensity: & St Dev (d.nm) \\
\hline Z.Average (d.nm): & 1349 & Peak 1: & 914,1 & 100,0 & 108,9 \\
\hline Pd: & 0.453 & Peak Z: & 0.000 & 0.0 & 0.000 \\
\hline Intercept: & 0.771 & Peak 3: & 0.000 & 0.0 & 0.000 \\
\hline
\end{tabular}
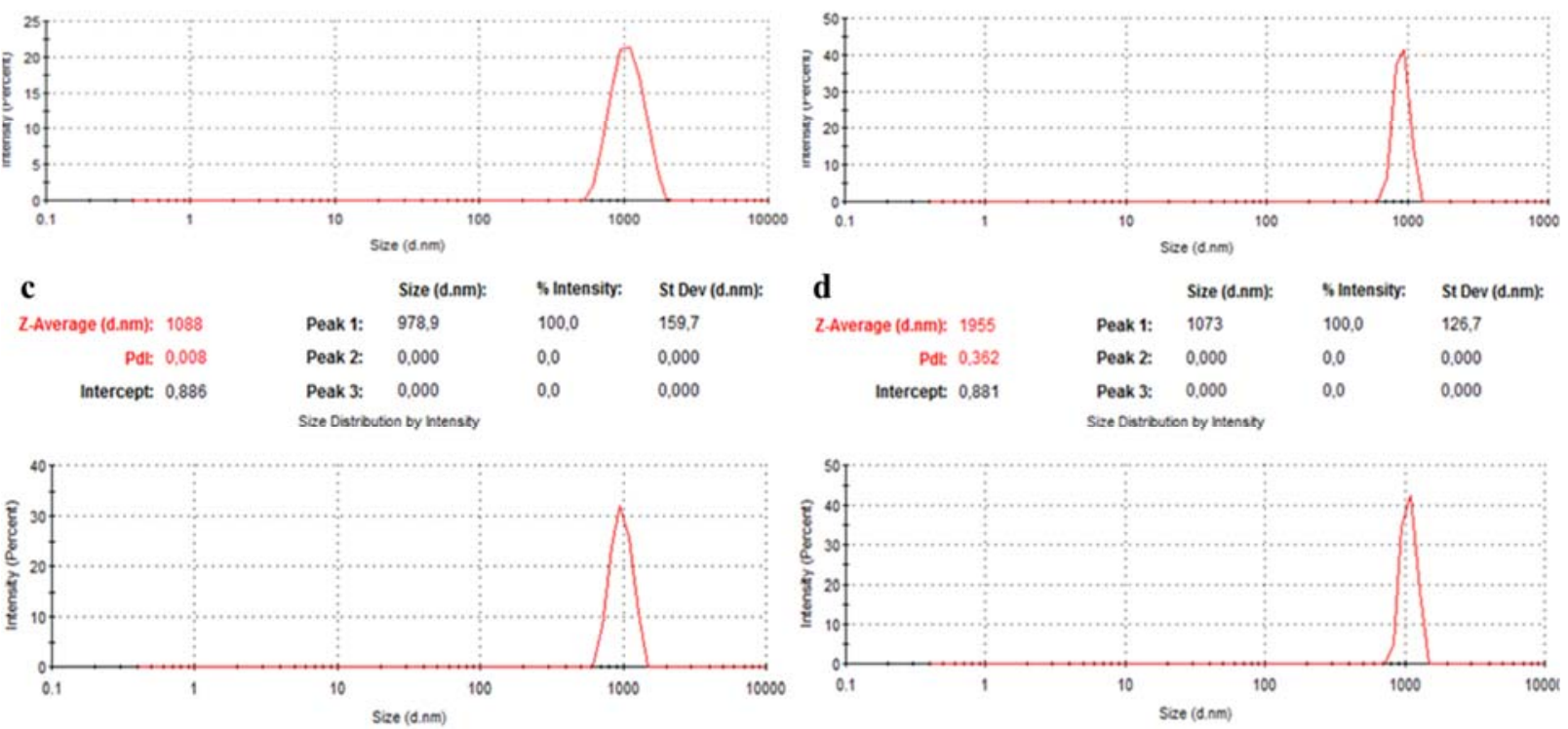

Figure 3. Particle size distribution of as-synthesized particles prepared with various molar ratios a) 1:1, b) 1:2, c) 1:3 and d) 1:4 OCCP:DADPM 
Experiments were performed for different reaction times (5, 10, 15, 20, 25, 30, 60, 90, 120, 150 and 180 $\mathrm{min}$ ) using the same procedure. When the SEM images for obtained spheres from $5 \mathrm{~min}$ to $30 \mathrm{~min}$ were compared, it was determined that size of particles were different from each other and changed in a range of $\cong 431-1204 \mathrm{~nm}$ (Figure 4). It was understood that during $30 \mathrm{~min}$, particle sizes increased, but did not change dramatically between $30 \mathrm{~min}$ and 3h (60 min:1123, 90 min:1154, 120 min:1034, 150 min:1097, $180 \mathrm{~min}: 1082 \mathrm{~nm}$ ) by SEM images and particle size distributions (Figure 4-6).

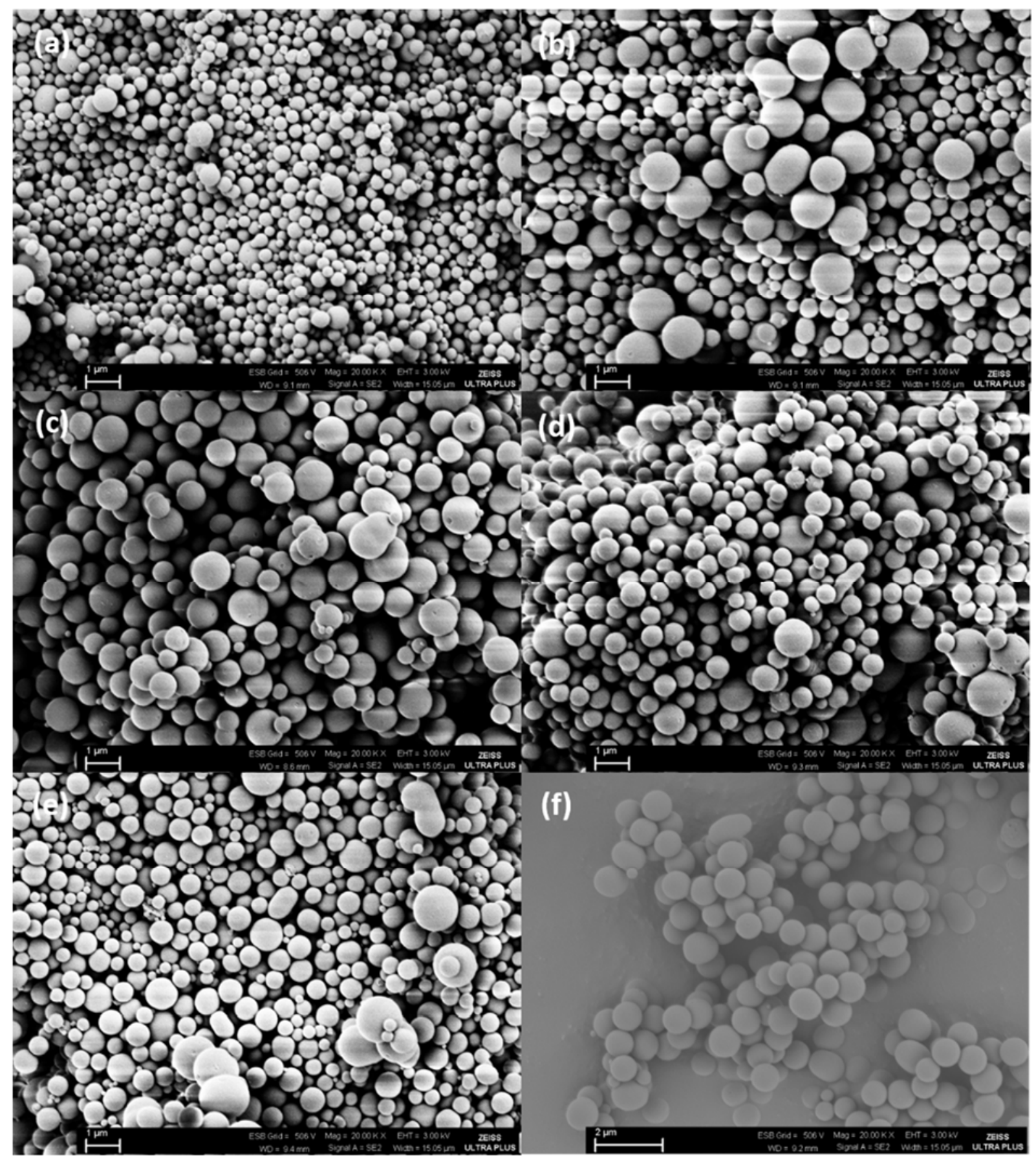

Figure 4. SEM images of (1:1/OCCP:DADPM) microspheres obtained in different reaction times of a) $5 \mathrm{~min}$ b) $10 \mathrm{~min} \mathrm{c)} 15 \mathrm{~min} \mathrm{d)} 20 \mathrm{~min}$ e) $25 \mathrm{~min} \mathrm{f)} 30 \mathrm{~min}$ 
Süzen and Metinoğlu Örüm / Anadolu Univ. J. of Sci. and Technology A-Appl. Sci. and Eng. 18 (5) - 2017

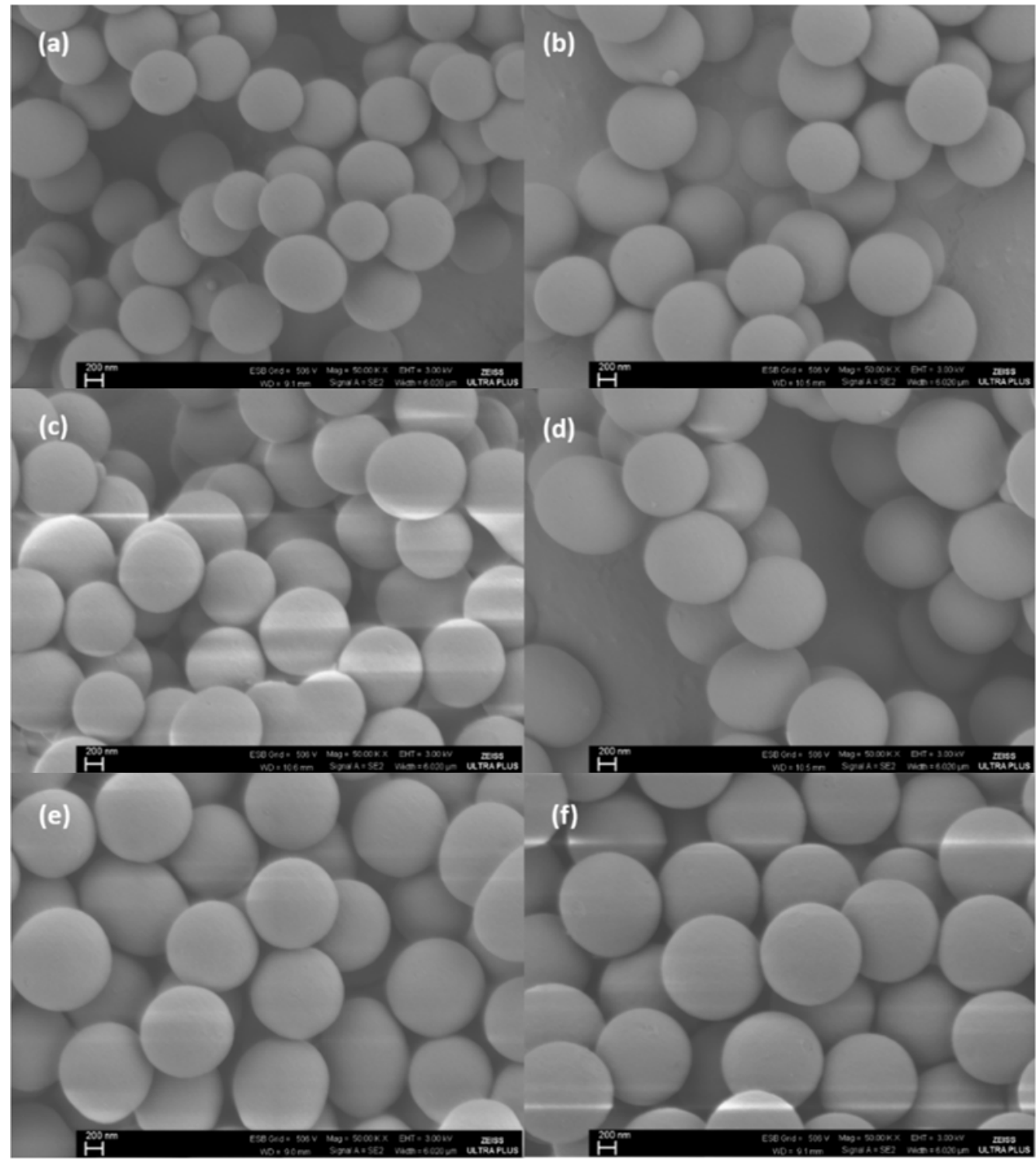

Figure 5. SEM images of microspheres obtained in different reaction times of (1:1/OCCP:DADPM) a) 30min b) $1 \mathrm{~h} \mathrm{c}) 1.5 \mathrm{~h} \mathrm{~d}$ ) $2 \mathrm{~h} \mathrm{e)} 2.5 \mathrm{~h} \mathrm{f}) 3 \mathrm{~h}$

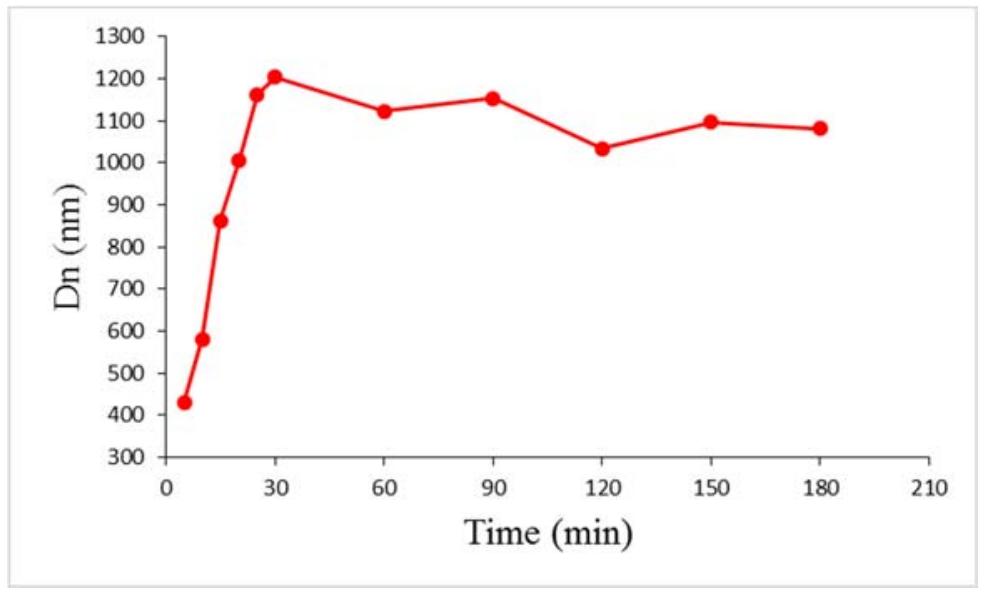

Figure 6. Effect of reaction time on diameter 
Besides, the effect of ultrasonic power on formation of polyphosphazene spheres was investigated. Experiments were performed at 35 and $53 \mathrm{kHz}$ ultrasonic power using (1:1) OCCP:DADPM molar ratio under same conditions. It is concluded that, morphologically worse polyphosphazene microspheres were obtained using $35 \mathrm{kHz}$ ultrasonic power (Figure 7). The particle sizes of formed particles under both 35 $\mathrm{kHz}$ and $53 \mathrm{kHz}$ ultrasonic power were approximately the same, $995 \mathrm{~nm}$ and $1082 \mathrm{~nm}$, respectively.

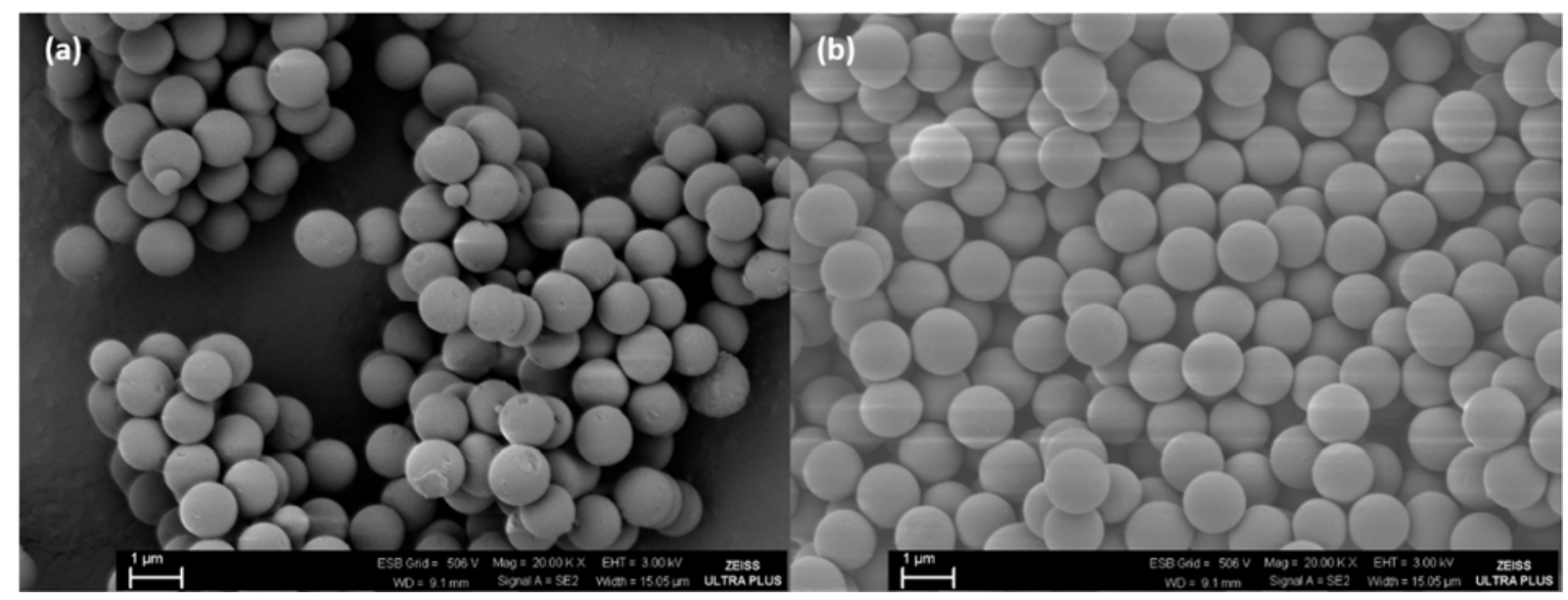

Figure 7. SEM images of (1:1) OCCP:DADPM microspheres prepared under different ultrasonic power a) $35 \mathrm{kHz}$ b) $53 \mathrm{kHz}$

Considering all the results, 3-hours reaction time, $53 \mathrm{kHz}$ ultrasonic power and (1:1) OCCP:DADPM molar ratio were found as the best experimental conditions.

It was understood from the results of reactivity experiments OCCP was a more reactive crosslinker than HCCP. It was also seen that the precipitation started within 5 minutes when OCCP was used as crosslinker in the reaction. (Figure 1.) However, difficultly in the precipitation was observed when HCCP was used. As seen in SEM images (Figure 8) and DLS measurements (Figure 9), smaller microspheres were obtained at the crosslinking reaction with DADPM, and particle sizes of synthesized microspheres with OCCP and HCCP were $1.082 \mu \mathrm{m}$ and $4.510 \mu \mathrm{m}$, respectively. From the results, it was proven that the OCCP is a better crosslinker than HCCP due to including more chlorine atoms than HCCP and its skeletal flexibility.

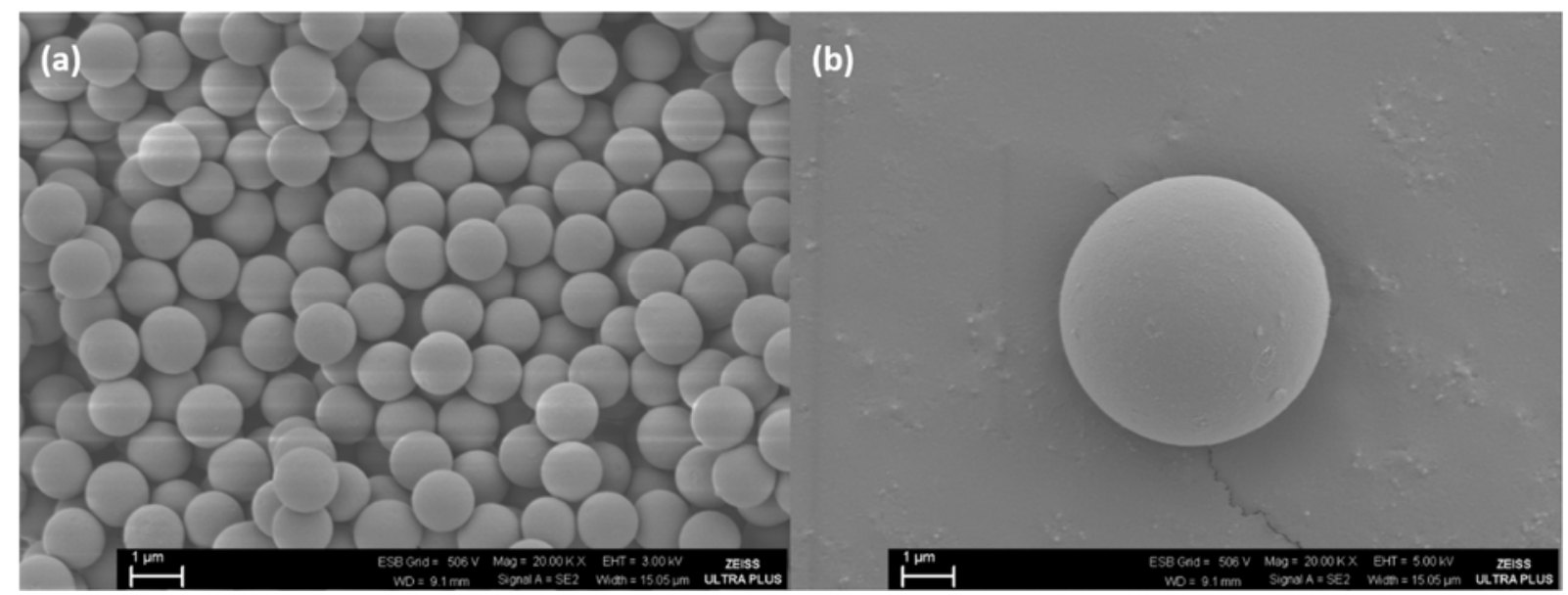

Figure 8. SEM images of microspheres using (a) OCCP, (b) HCCP as crosslinkers 
a

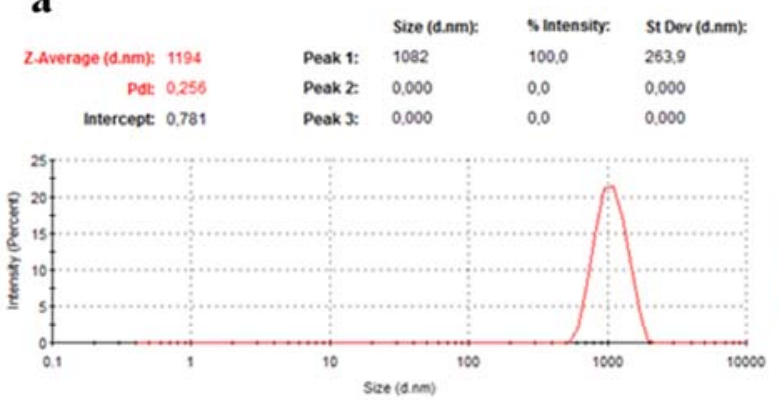

b

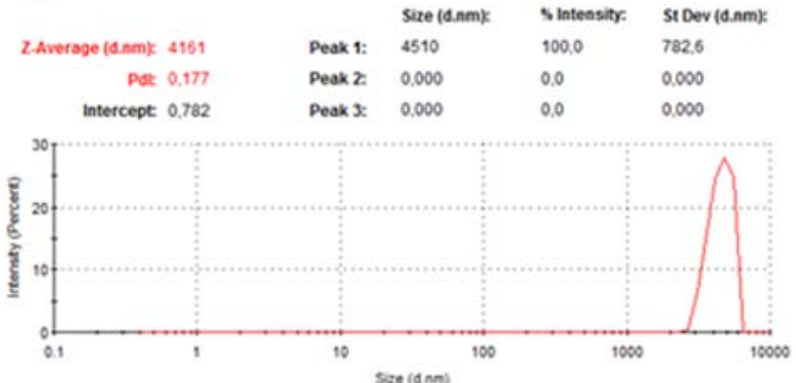

Figure 9. The particle size distributions of microspheres using (a) OCCP, (b) HCCP as crosslinkers

\subsection{Structural Characterization of OCDA-MS}

The functional groups on the chemical structure of the resulting OCDA-MS (1:1 OCCP:DADPM) were characterized by FTIR. N-H stretching and bending bands indicating the existence of primary amine groups, due to nucleophilic substitution over $\mathrm{NH}_{2}$ - groups of DADPM, on the OCDA-MS were appeared at 3362-3075 and $1513 \mathrm{~cm}^{-1}$, respectively. The absorption band at $3028 \mathrm{~cm}^{-1}$ was attributed to the aromatic $\mathrm{C}-\mathrm{H}$ stretching vibration of aromatic rings. Aliphatic $\mathrm{C}-\mathrm{H}$ stretching vibration bands can be seen at 2907 and $2833 \mathrm{~cm}^{-1}$. Moreover, the characteristic $\mathrm{P}=\mathrm{N}$ and $\mathrm{P}-\mathrm{N}$ absorption bands of OCCP were observed at 1293,1228 and $963 \mathrm{~cm}^{-1}$, respectively. The asymmetric and symmetric vibrations of $\nu_{\mathrm{PCl} 2}$ are observed for OCCP in the ranges of $601-574 \mathrm{~cm}^{-1}$ and $523-517 \mathrm{~cm}^{-1}$. The weak P-Cl band at $497 \mathrm{~cm}^{-1}$ showed that microspheres still contain chlorine atoms in small quantities after polycondensation (Figure 10). Overlapped FTIR spectra of OCDA-MS, OCCP and DADPM were shown for comparison (Figure 11). Obviously, FTIR spectrum of OCDA-MS contain characteristic bands which are expected from the polymeric structure synthesized from OCCP and DADPM molecules. Thus, the self-assembly polycondensation reaction between OCCP and DADPM has been successfully achieved.

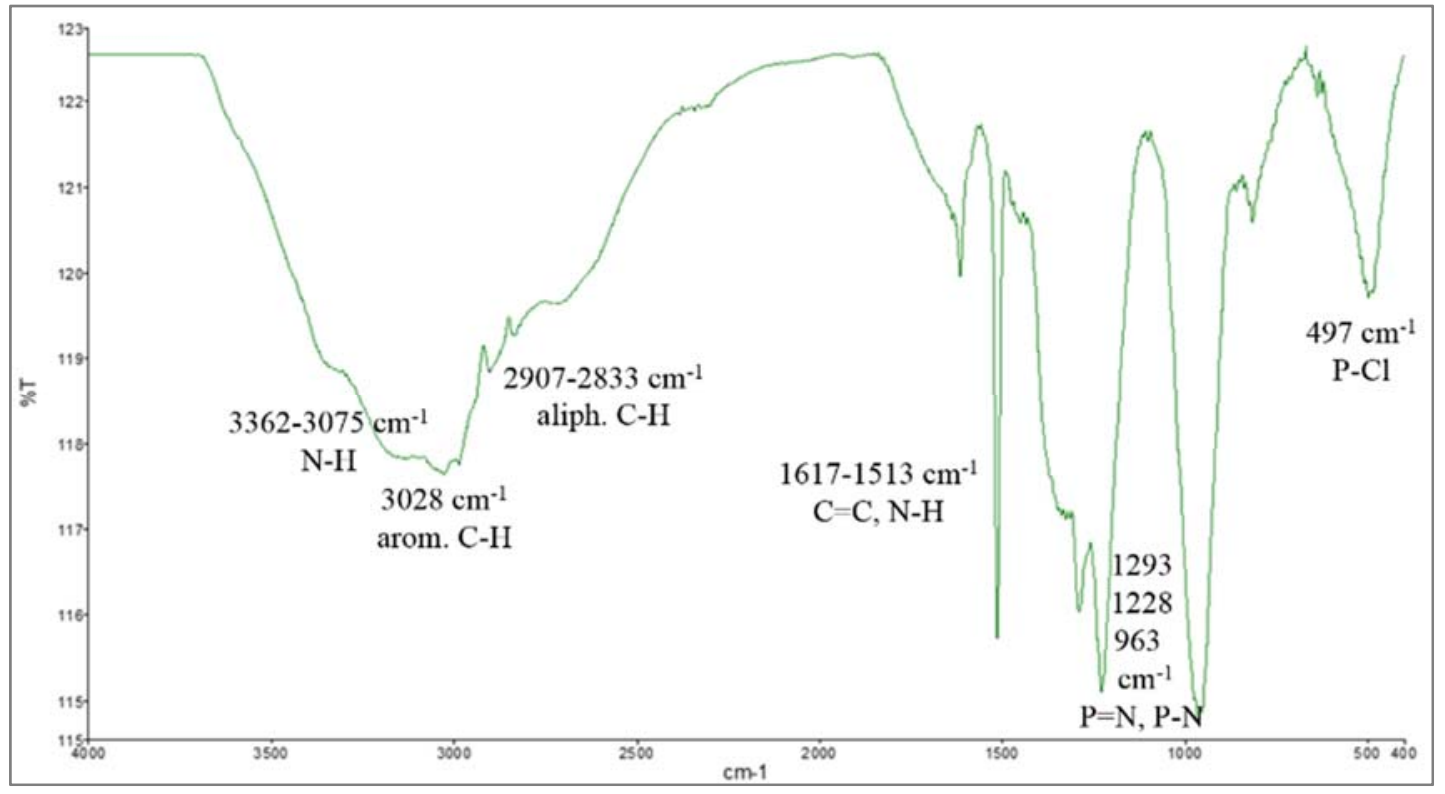

Figure 10. FTIR spectra of OCDA-MS 


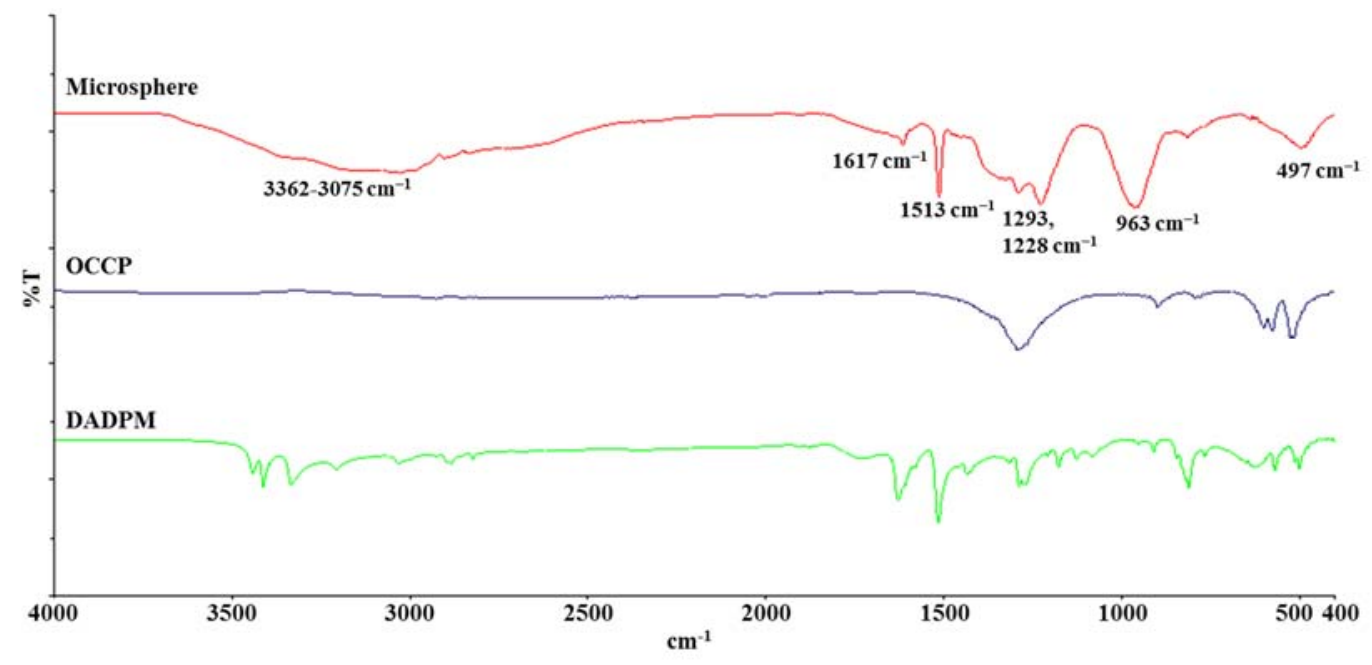

Figure 11. FTIR spectra of OCDA-MS, OCCP and DADPM

The thermal stability of OCDA-MS obtained from the precipitation polymerization was also characterized by TGA in air, as shown in Figure 12. It is clear that OCDA-MS has thermal stability compared with OCCP and DADPM due to consisted of fully cross-linked cyclic structures. The mass loss is through desorption of adsorbed water and solvent below $100^{\circ} \mathrm{C}$. The mass loss of DADPM occured in two steps, $74.65 \%$, between $162.6-377.4{ }^{\circ} \mathrm{C}$ and $24.12 \%$ between $377.4{ }^{\circ} \mathrm{C}-751.8{ }^{\circ} \mathrm{C}$. The fully cross-linked phosphazene rings of OCDA-MS and organic monomer (DADPM) bonded to phosphazene rings have decomposed $73.64 \%$ from $394{ }^{\circ} \mathrm{C}$ to $837{ }^{\circ} \mathrm{C}$.

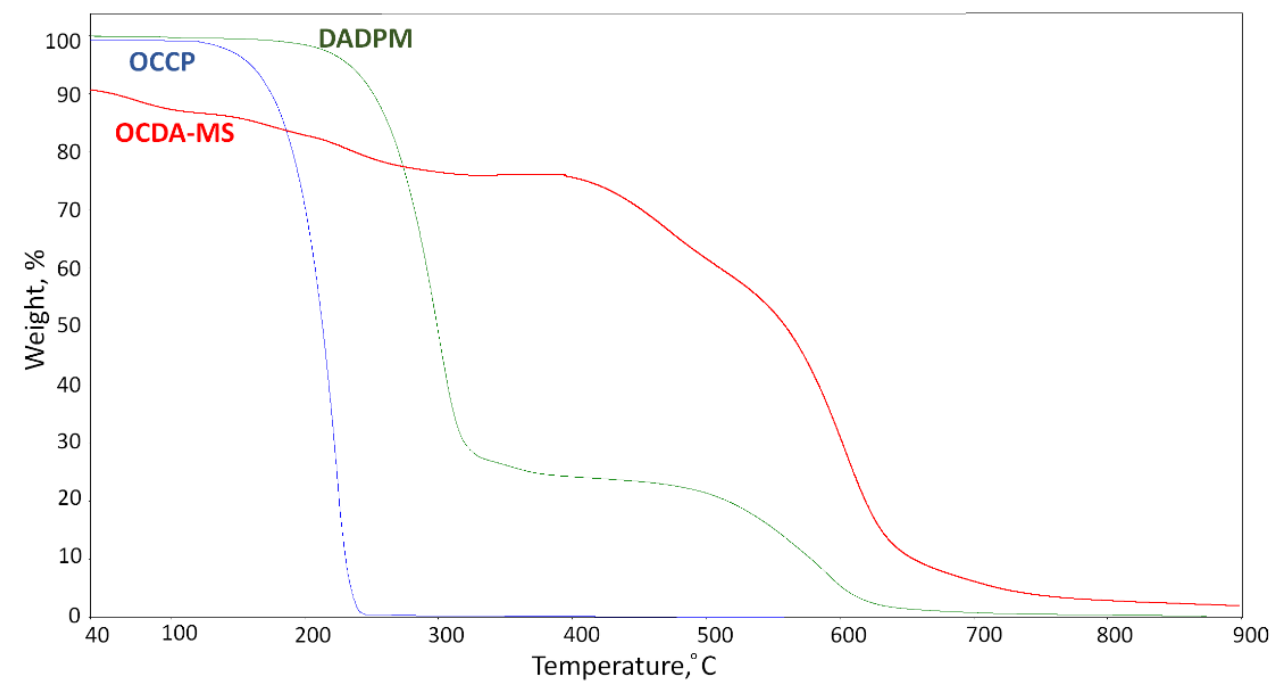

Figure 12. TGA curves of OCCP, DADPM and OCDA-MS in air

The XRD pattern of OCDA-MS is shown in Figure 13. The XRD pattern of the powder OCDA-MS indicated a broad diffraction peak at about $20^{\circ}$ corresponding typical amorphous structure. It was understood from XRD pattern that cyclomatrix structure succesfully formed [25, 31, 32]. Besides, pattern doesn't have any peak belonging the TEA.HCl salt obtained during the polycondensation reaction, showing OCDA-MS were purified from the residue with water very well. 


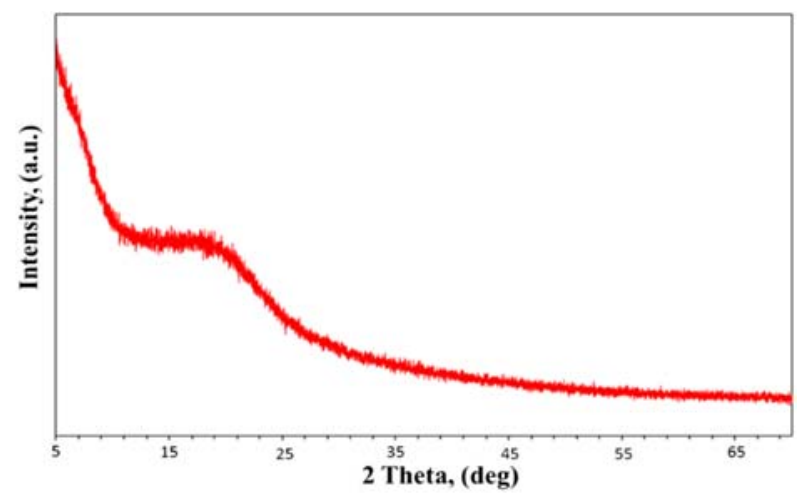

Figure 13. XRD pattern of OCDA-MS (1:1/OCCP:DADPM)

In addition, UV-vis and fluorescence properties of OCDA-MS were investigated. The UV absorption and fluorescence emission spectra of dispersed OCDA-MS in methanol are shown in Figure 14. OCDAMS and DADPM displayed absorption peaks at 262 and $290 \mathrm{~nm}$, respectively, and OCDA-MS have no absoption in the visible region (Figure 14a). OCDA-MS which have amine groups on surface and benzene ring in cyclomatrix structure displayed a fluorescence emission peak at $485 \mathrm{~nm}$ when excited with $240 \mathrm{~nm}$ light due to $\pi$-conjugation in the cyclomatrix polymeric structure (Figure 14b).
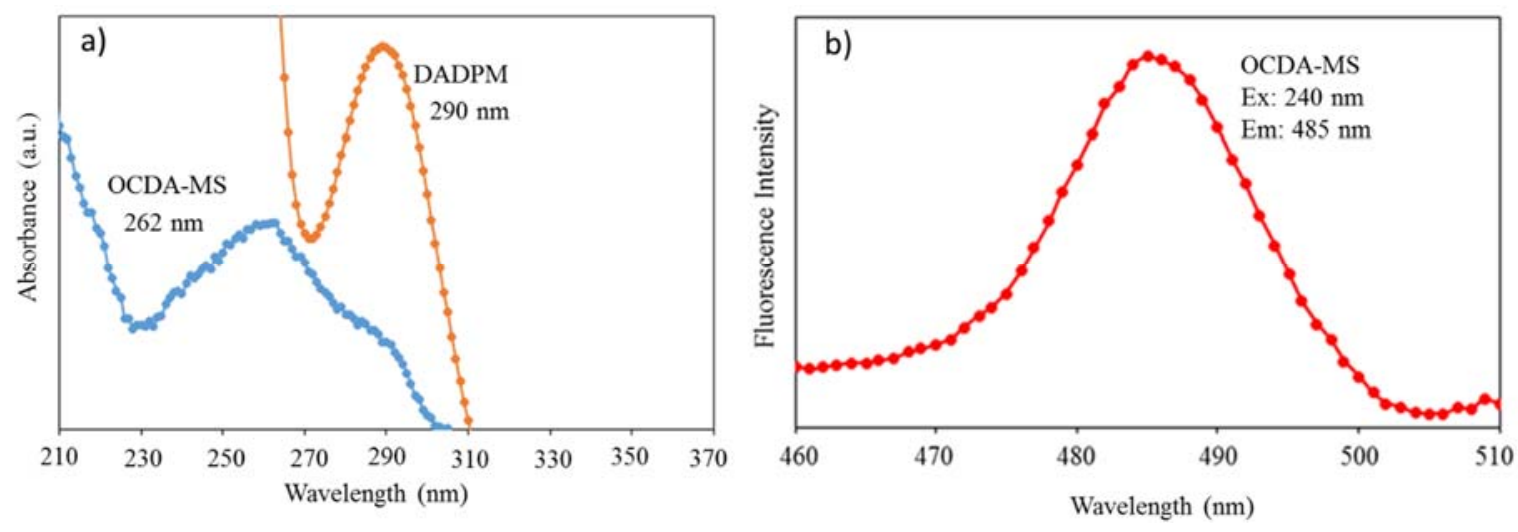

Figure 14. a) UVspectra of OCDA-MS and DADPM, b) fluorescence spectra of OCDA-MS

\section{CONCLUSION}

In conclusion, novel inorganic-organic hybride cyclomatrix type polyphosphazene-based microspheres were developed using new crosslinker molecules, OCCP and DADPM as monomers by self-assembly polycondensation reaction approach. The results showed that in this type of synthesis reactions, OCCP can be used as a substitute of HCCP as a good alternative and effective crosslinker molecule. Different molar ratios of the reactants, reaction time and ultrasonic power were investigated on preparing morphologically good spheres. The best molar ratio was determined as 1:1 (OCCP:DADPM) between different molar ratios from the SEM images. Besides, it was also found that when reaction time was changed from 5 min to 3 hours, size of OCDA-MS ranged from 0.43 to $1.00 \mu \mathrm{m}$. Morphologically better microspheres were prepared by $53 \mathrm{kHz}$ ultrasonic power. FTIR spectrum demonstrated that the formation of the microspheres were achieved succesfully in comparison to FTIR spectra of OCCP and DADPM molecules. OCDA-MS indicated UV and fluorescent properties due to $\pi$-conjugation of aromatic rings in the polymeric structure. XRD spectrum showed that microspheres have characteristic amorphous structure. Thermal decomposition of microspheres occured up to $837{ }^{\circ} \mathrm{C}$. Considering their good surface and other properties, synthesized polyphosphazene based microspheres are expected to be useful materials for various practical applications, such as, heavy metal/dye adsorption, drug release and catalysis. 


\section{ACKNOWLEDGEMENTS}

The authors acknowledge to the "Anadolu University, Scientific Research Unit" Grant No. 1506F536 for their financial support.

\section{REFERENCES}

[1] Köhler J, Kühl S, Keul H, Möller M, Pich A. Synthesis and Characterization of Polyamine-Based Cyclophosphazene Hybrid Microspheres. J Polym Sci Pol Chem 2014, 52: 527-536.

[2] Wang Y, Mu J, Li L, Shi L, Zhang W, Jiang Z. Preparation and properties of novel fluorinated crosslinked polyphosphazene micro-nano spheres. High Perform Polym 2012, 24(3): 229-236.

[3] Mohanty P, Landskron K. Simple systematic synthesis of size-tunable covalent organophosphonitridic framework nano- and microspheres. New J Chem 2010, 34: 215-220.

[4] Zhao Z, Ji J. Synthesis and Tribological Behaviors of Epoxy/Phosphazene-Microspheres Coatings under Dry Sliding Condition. Adv Eng Mater 2014, 16(8): 988-995.

[5] Ozay H, Ozay O. Synthesis and characterization of drug microspheres containing phosphazene for biomedical applications. Colloids and Surfaces A: Physicochem. Eng. Aspects 2014, 450: 99-105.

[6] Wei W, Huang X, Chen K, Tao Y, Tang X. Fluorescent organic-inorganic hybrid polyphosphazene microspheres for the trace detection of nitroaromatic explosives. RSC Adv 2012, 2: 3765-3771.

[7] Fu J, Wang M, Zhang C, Zhang P, Xu Q. High hydrogen storage capacity of heteroatom-containing porous carbon nanospheres produced from cross-linked polyphosphazene nanospheres. Mater Lett 2012, 81: 215-218.

[8] Wang D, Hu Y, Meng L, Wang X, Lu Q. One-pot synthesis of fluorescent and cross-linked polyphosphazene nanoparticles for highly sensitive and selective detection of dopamine in body fluids. RSC Adv 2015, 5: 92762-92768.

[9] Pan T, Huang X, Wei H, Wei W, Tang X. Intrinsically Fluorescent Microspheres with Superior Thermal Stability and Broad Ultraviolet-Visible Absorption Based on Hybrid Polyphosphazene Material Macromol Chem Phys 2012, 213: 1590-1595.

[10] Wang M, Fu J, Chen Z, Wang X, Xu Q. In situ growth of gold nanoparticles onto polyphosphazene microspheres with amino-groups for alcohol oxidation in aqueous solutions. Mater Lett 2015, 143: 201-204.

[11] Tian Z, Hess A, Fellin CR, Nulwala H, Allcock HR. Phosphazene High Polymers and Models with Cyclic Aliphatic Side Groups: New Structure-Property Relationships. Macromolecules 2015, 48: 4301-4311.

[12] Modzelewski T, Wilts E, Allcock HR. Elastomeric Polyphosphazenes with PhenoxyCyclotriphosphazene Side Groups. Macromolecules 2015, 48: 7543-7549.

[13] Chen K, Huang X, Wan C, Liu H. Heteroatom-doped mesoporous carbon nanofibers based on highly cross-linked hybrid polymeric nanofibers: Facile synthesis and application in an electrochemical supercapacitor. Mater Chem Phys 2015, 164: 85-90. 
[14] Jin W, Yuan L, Liang G, Gu A, Multifunctional Cyclotriphosphazene/Hexagonal Boron Nitride Hybrids and Their Flame Retarding Bismaleimide Resins with High Thermal Conductivity and Thermal Stability. Appl Mater Interfaces 2014, 6: 14931-14944.

[15] Süzen Y, Metinoğlu S. Synthesis and characterization of novel inorganic and organic hybrid poly[cyclotriphosphazene-co-(4,4'-diaminodiphenylmethane)] microspheres via one-pot selfassembly polycondensation approach. J Turkish Chem Soc Sect Chem (JOTCSA) 2016, 3 (2): 167182.

[16] Beşli S, Mutlu C, İbişoğlu H, Yüksel F, Allen CW. Synthesis of a New Class of Fused Cyclotetraphosphazene Ring Systems. Inorg Chem 2015, 54: 334-341.

[17] Liu X, Breon JP,Chen C,Allcock HR. Substituent exchange reactions of trimeric and tetrameric aryloxycyclophosphazenes with sodium 2,2,2-trifluoroethoxide. Dalton. Trans 2012, 41: 2100-2109.

[18] Zhu X, Liang Y, Zhang D, Wang L,Ye Y, Zhao Y. Synthesis and Characterization of Side GroupModified Cyclotetraphosphazene Derivatives. Phosphorus Sulfur Silicon Relat Elem 2011,186: 281286.

[19] Krause WE, Parvez M, Visscher KB, Allcock HR. Synthesis and Structure of AdamantaneContaining Phosphazenes. Inorg Chem 1996, 35: 6337-6338.

[20] Paasch S, Krüger K, Thomas B. Solid-state nuclear magnetic resonance investigations on chlorocyclophosphazenes. Solid State Nucl Mag 1995, 4: 267280.

[21] Beşli S, İbişoğlu H, Kılıç A, Ün İ, Yüksel F. Spiro, ansa-derivatives of cyclotetraphosphazenes with a tetrafluorobutane-1,4-diol. Polyhedron 2010, 29(17): 3220-3228.

[22] Huang Z, Chen S, Lu X, Lu Q. Water-triggered self-assembly polycondensation for the one-pot synthesis of cyclomatrix polyphosphazene nanoparticles from amino acid ester. Chem Commun 2015, 51: 8373-8376.

[23] Zhang JX, Qiu LY, Wu XL, Jin Y, Zhu KJ. Temperature-Triggered Nanosphere Formation Through Self-Assembly of Amphiphilic Polyphosphazene. Macromol Chem Phys 2006, 207(14): 1289-1296.

[24] Zhang P, Huang X, Fu J, Huang Y, Zhu Y, Tang X. A One-Pot Approach to Novel Cross-Linked Polyphosphazene Microspheres with Active Amino Groups. Macromol Chem and Phys 2009,210: $792-798$.

[25] Wei W, Lu R, Ye W, Sun J, Zhu Y, Luo J, Liu X. Liquid Marbles Stabilized by Fluorine-Bearing Cyclomatrix Polyphosphazene Particles and Their Application as High-Efficiency Miniature Reactors. Langmuir 2016, 32(7): 17071715.

[26] Chen C, Zhu X, Gao Q, Fang F, Wang L, Huang X. Immobilization of lipase onto functional cyclomatrix polyphosphazene microspheres. J Mol Catal B-Enzym 2016, 132: 67-74.

[27] Fu J, Chen Z, Wu X, Wang M, Wang X, Zhang J, Zhang J, Xu Q. Hollow poly(cyclo triphosphazene-co-phloroglucinol) microspheres: An effective and selective adsorbent for the removal of cationic dyes from aqueous solution. Chem Eng J 2015, 281: 42-52. 
[28] Liu W, Huang X, Wei H, Chen K, Gao J, Tang X. Facile preparation of hollow crosslinked polyphosphazene submicrospheres with mesoporous shells. J Mater Chem 2011, 21(34): 1296412968.

[29] Zhu Y, Fu J, Zhu L, Tang X, Huang X. Preparation of novel hybrid inorganic-organic hollow microspheres via a self-template approach. Polym Int 2008, 57(3): 449453.

[30] Chang F, Huang X, Wei H, Chen K, Shan C, Tang X. Intrinsically fluorescent hollow spheres based on organic-inorganic hybrid polyphosphazene material: Synthesis and application in drug release. Mater Lett 2014, 125: 128131.

[31] Fu J, Wang M, Zhang C, Wang X, Wang H, Xu Q. Template-induced covalent assembly of hybrid particles for the facile fabrication of magnetic $\mathrm{Fe} 3 \mathrm{O} 4-$ polymer hybrid hollow microspheres. J Mater Sci 2013, 48(9): 3557-3565.

[32] Hu Y, Meng L, Niu L, Lu Q. Highly Cross-Linked and Biocompatible Polyphosphazene-Coated Superparamagnetic $\mathrm{Fe}_{3} \mathrm{O}_{4}$ Nanoparticles for Magnetic Resonance Imaging. Langmuir 2013, 29(29): 9156-9163.

[33] Tang X, Huang X. Modern Inorganic Synthetic Chemistry, Elsevier, AMSTERDAM, 2011. 